\title{
A Reply to Towell's Book Review of Neural Network Perception for Mobile Robot Guidance
}

\author{
DEAN A. POMERLEAU \\ School of Computer Science, Carnegie Mellon University, Pittsburgh, PA 15213
}

First, let me thank Geoff Towell for his comprehensive critique of my book, "Neural Network Navigation for Mobile Robot Guidance".

His most significant criticism of the research described in the book is the lack of thorough testing and evaluation. The book covers a lot of research ground, and I agree that in some places the coverage in not as detailed or quantitative as ideally one would like. The primary reason for this shortcoming is the logistical difficulty of conducting controlled tests of a system designed to operate in an uncontrolled, real world environment. For example, to perform the "water drop" experiment the reviewer mentions, I spent an entire day on my hands and knees crawling after the vehicle measuring its position and digging through leaves on the road to find its true center.

Fortunately, several developments since the book was completed are allowing us to address these evaluation issues more thoroughly. the reviewer specifically criticizes the claim that trained ALVINN networks are insensitive to variations in lighting and weather conditions. Admittedly the evidence for this in the book is limited. However last summer we conducted a more compelling test similar in many respects to the "panel of judges" experiment suggested by the reviewer. In early spring we ported ALVINN to the vehicle of one of our research partners, Martin Marietta Corporation, and taught them to use it. They then trained two networks, one for a two lane road with lane markings and a second for a single lane dirt road. They proceeded to use these networks for nearly two months of day-in, day-out testing without retraining. These tests culminated in a two day demonstration of ALVINN driving several times over a $3 / 4$ mile course in front of over 200 people from academia, industry and the government. Many of these observers had initially been skeptical of ALVINN's capabilities, but all came away from the test impressed with its performance. While still anecdotal, we think this experiment provides fairly convincing evidence of ALVINN's ability to cope with changing conditions.

In another related development, Carnegie Mellon has recently been awarded a grant from the National Highway Traffic Safety Administration (NHTSA) to quantitatively evaluate the performance of ALVINN and several other systems as road way departure warning devices. Single vehicle roadway departures crashes, typically caused by driver fatigue or inattention, account for over 13,000 driving fatalities each year in this country. We believe ALVINN could prevent a significant fraction of these accidents by either warning a driver, or assuming temporary control, when the vehicle starts to drift off the roadway. 
An effective crash prevention system must work reliably under all weather and lighting conditions, and a major portion of the NHTSA project involves verifying ALVINN's capabilities. As part of this project, we are developing the techniques and acquiring the facilities necessary to carefully evaluate ALVINN's performance. One of these new developments is a downward looking lane marker tracker, that can estimate in real time the vehicle's lateral position to within several centimeters. This system only works when there are clearly visible lane markings, but under these conditions it should allow us to carefully measure ALVINN's road following performance.

Overall, the ALVINN system continues to evolve and improve. The book "Neural Network Navigation for Mobile Robot Guidance" provides a comprehensive snapshot of the system at one point during this process. Much of our current ALVINN research addresses the shortcomings the reviewer identifies, particularly related to quantitative evaluation.

Received and Accepted April 25, 1994 